\title{
Laser Ablation of a Nonlesional Cingulate Gyrus Epileptogenic Zone Using Robotic-Assisted Stereotactic EEG Localization: A Case Report
}

\author{
Pavan S. Upadhyayula ${ }^{\mathrm{a}}$ Robert C. Rennert ${ }^{\mathrm{a}}$ Reid Hoshide ${ }^{\mathrm{a}}$ Shifteh Sattar ${ }^{\mathrm{b}}$ David D. Gonda ${ }^{\mathrm{a}}$ \\ a Department of Neurosurgery, University of California San Diego, San Diego, CA, USA; \\ ${ }^{b}$ Department of Neurosciences, University of California San Diego, San Diego, CA, USA
}

\section{Keywords}

Stereotactic EEG · Laser ablation .

Robotic-assisted surgery $\cdot$ ROSA

\begin{abstract}
Background: Nonlesional cingulate gyrus epilepsy is rare, difficult to diagnose, and challenging to treat. Methods: We report the use of ROSA (Medtech Surgical, Inc., New York, NY, USA) robotic assistance for stereotactic EEG (S-EEG) localization and therapeutic thermal laser ablation of a nonlesional cingulate gyrus epileptogenic zone in a 17-year-old female with intractable partial epilepsy. Results: After an inconclusive exhaustive initial workup, robotic-assisted S-EEG localized the patient's seizure focus to the right cingulate gyrus. Robotic-assisted lesioning of the cingulate gyrus was performed via 5 total ablations with 3 minimally invasive catheters. There were no perioperative complications. The patient was discharged home on postoperative day 2 at her neurologic baseline. She was seizure free for 8 months postoperatively, with a sustained partial response through the 23-month follow-up. Conclusions: This report expands the technical uses, pathologies, and patient populations being treated via robotic-assisted neurosurgery.
\end{abstract}

(c) 2019 S. Karger AG, Basel

\section{KARGER}

(๑) 2019 S. Karger AG, Basel

E-Mail karger@karger.com

www.karger.com/sfn

\section{Introduction}

Medically intractable epilepsy is associated with high rates of psychiatric and physical morbidity [1], as well as an increased risk of sudden unexplained death [2]. Surgical resection or ablation of epileptogenic zones (EZ) can potentially cure intractable epilepsy, with preoperative localization of EZ traditionally limited by a trade-off in anatomical accuracy for monitoring risk. Mesial or deep brain EZ can nonetheless be challenging to localize with noninvasive techniques, including scalp electroencephalography (EEG), functional magnetic resonance imaging (fMRI), positron emission tomography (PET), and magnetoencephalography (MEG) [3], and often require more invasive approaches such as subdural grid EEG electrodes [4].

Cingulate gyrus epilepsy has distinct semiological, clinical, and anatomical features that make diagnosis and surgical cure particularly challenging. Anatomically, the cingulate gyrus overlies the superior convexity of the corpus callosum, allowing aberrant unilateral focal electrical activity to spread quickly and create diffuse, nonlocalizable seizure activity in the frontal and parietal lobes. Close connectivity to the limbic system can also complicate seizure localization in this region, as auras of déjà vu, fear, or laughter followed by ictal hyperkinetic behavior
Prof. David D. Gonda, MD

University of California San Diego

7910 Frost St., Suite 120

San Diego, CA 92123 (USA)

E-Mail dgonda@ucsd.edu 
commonly seen with cingulate gyrus epilepsy can be mistaken for nonepileptic events or temporal/frontal lobe seizures [5]. As such, only a handful of reported cases describe localization and resection of cingulate gyrus EZ [6-9], the majority of which report lesional EZ secondary to malignancy, dysplastic lesions, or generalized parenchymal volume loss visible on conventional MRI $[6,8,9]$.

Reports of surgical treatment of imaging-negative, nonlesional cingulate gyrus epilepsy are especially rare due to historical challenges in EZ localization in these cases. Specifically, interhemispheric grid EEG is technically challenging in cingulate gyrus epilepsy due to the depth of electrode placement and difficulty in cleanly separating the pia of reciprocal cingulate gyri. The development of stereotactic depth electrode EEG (S-EEG) has improved the ability to safely identify EZ in pediatric patients with difficult to localize refractory epilepsy, and the utility of this approach for localization of nonlesional cingulate gyrus EZ has been demonstrated in limited reports $[8,10]$.

Even after a cingulate gyrus EZ is identified, however, surgical options have traditionally been poor. While resection of surgically accessible EZ localized via S-EEG can lead to seizure freedom in approximately $55 \%$ of cases [11], resection of cingulate gyrus EZ is difficult without disrupting critical nearby structures including the supplementary motor area anteriorly and the precuneus posteriorly. As such, morbidity rates with this procedure are more than $40 \%$, with a nearly $10 \%$ risk of permanent neurologic injury [6].

Robotic-assisted (ROSA, Medtech Surgical, Inc.) stereotactic laser ablation of EZ is an emerging minimally invasive technique for the treatment of eloquent $\mathrm{EZ}$ in both adult and pediatric populations [12]. In this report, we describe the robotic-assisted S-EEG localization and laser ablation of a nonlesional cingulate gyrus EZ in a pediatric patient.

\section{Case Report}

\section{History}

The patient is a 17-year-old female with intractable partial epilepsy diagnosed at 8 years of age. Her seizures occurred mainly when sleeping, often multiple times per night, and caused her to awaken with sensations of intense fear, and occasional hypermotor movements, urinary incontinence, and somniloquy. These seizures lasted between 10 and $45 \mathrm{~s}$, and significantly disrupted the patient's sleep. Daytime seizures occurred less frequently and were associated with an aura of intense fear. The patient's seizures were successfully treated for a 3-year period with phenytoin, but ultimately became refractory to multiple antiepileptic drug (AED) therapy including levetiracetam, oxcarbazepine, lacosamide, clobazam, and clonazepam. The patient's family history was notable for a maternal grandmother with lifelong epilepsy and a maternal great uncle with grand mal seizures while in high school. The patient's interictal neurologic exam was normal, and neuropsychological testing was largely above average.

\section{Workup}

An exhaustive neurology workup including multiple video scalp EEG, MRI scan, fMRI, PET, and MEG, all failed to definitively localize the patient's seizures, but was suggestive of a frontal region origin (Fig. 1, 2). Genetic testing was notable for a DEPDC5 mutation seen in patients with autosomal dominant familial focal epilepsy with variable foci and sometimes associated with imaging-negative cortical dysplasia [4].

\section{Operations}

The patient subsequently underwent invasive monitoring with S-EEG. Electrodes were placed by ROSA robotic guidance (Medtech Surgical Inc.), with emphasis on the cingulate gyrus and mesial aspects of the superior and inferior frontal lobes. Seven electrodes were placed on the left and 6 on the right (to confirm laterality), for a total of 13 electrodes (Fig. 3). S-EEG recording captured 13 typical seizures and intervening interictal periods over the course of 3 days. Interictal data revealed slowing in the right cingulate gyrus (covered by 3 electrodes; A, B, and C moving anterior to posterior), left anterior-superior frontal gyrus, and left orbitofrontal region consistent with cerebral dysfunction in these areas. Recurrent epileptiform discharges were also seen in the mid-right cingulate gyrus (electrode C) and left anterior-superior frontal gyrus, with recurrent paroxysmal fast activity noted in the right anterior cingulate gyrus (electrode A); these data signified potential epileptogenicity in the left superior frontal gyrus and right cingulate gyrus. Ictal data nonetheless revealed electroclinical seizures originating from the right anterior cingulate gyrus (electrode A), with almost immediate involvement of the left anterior-superior frontal gyrus.

Extraoperative functional mapping was then performed, with successful provocation of typical seizures from stimulation of S-EEG electrode $\mathrm{A}$ in the anterior right cingulate gyrus, and generation of typical auras in decreasing intensity (but no seizures) with stimulation of leads $\mathrm{B}$ and $\mathrm{C}$ moving posteriorly within the right cingulate gyrus. Stimulation of the contralateral side did not result in typical auras or seizures. Given this precise localization of the EZ, stereotactic laser ablation of the anterior portion of the right cingulate gyrus with robotic guidance was performed in a subsequent procedure.

Three Visualase catheters (Medtronic, Inc.) for laser ablation were placed in the operating room in the following areas: the anterior-most portion of the cingulate gyrus as it wrapped around the genu of the corpus callosum, the superior portion of the cingulate gyrus as it passed above the genu of the corpus callosum, and within the cingulate gyrus along the most dorsal aspect of the corpus callosum (Fig. 4). The patient was then transferred to the MRI scanner for confirmatory imaging and ablation. Ablation was performed according to the manufacturer's instructions, at $\sim 65 \%$ power and with safety markers lining the periphery of the ablation site to ensure no heat was transferred to the corticospinal tracts, the corpus callosum, or the contralateral cingulate gyrus. Three ablations were performed with the anterior-most catheter, separated by $8 \mathrm{~mm}$ of retraction along the catheter trajectory. Two ablations were performed with each of the remaining catheters in a similar fashion. Diffusion weighted imaging was used to confirm complete ablation of the anterior right cingulate gyrus (Fig. 5) before the uneventful removal of all catheters. 


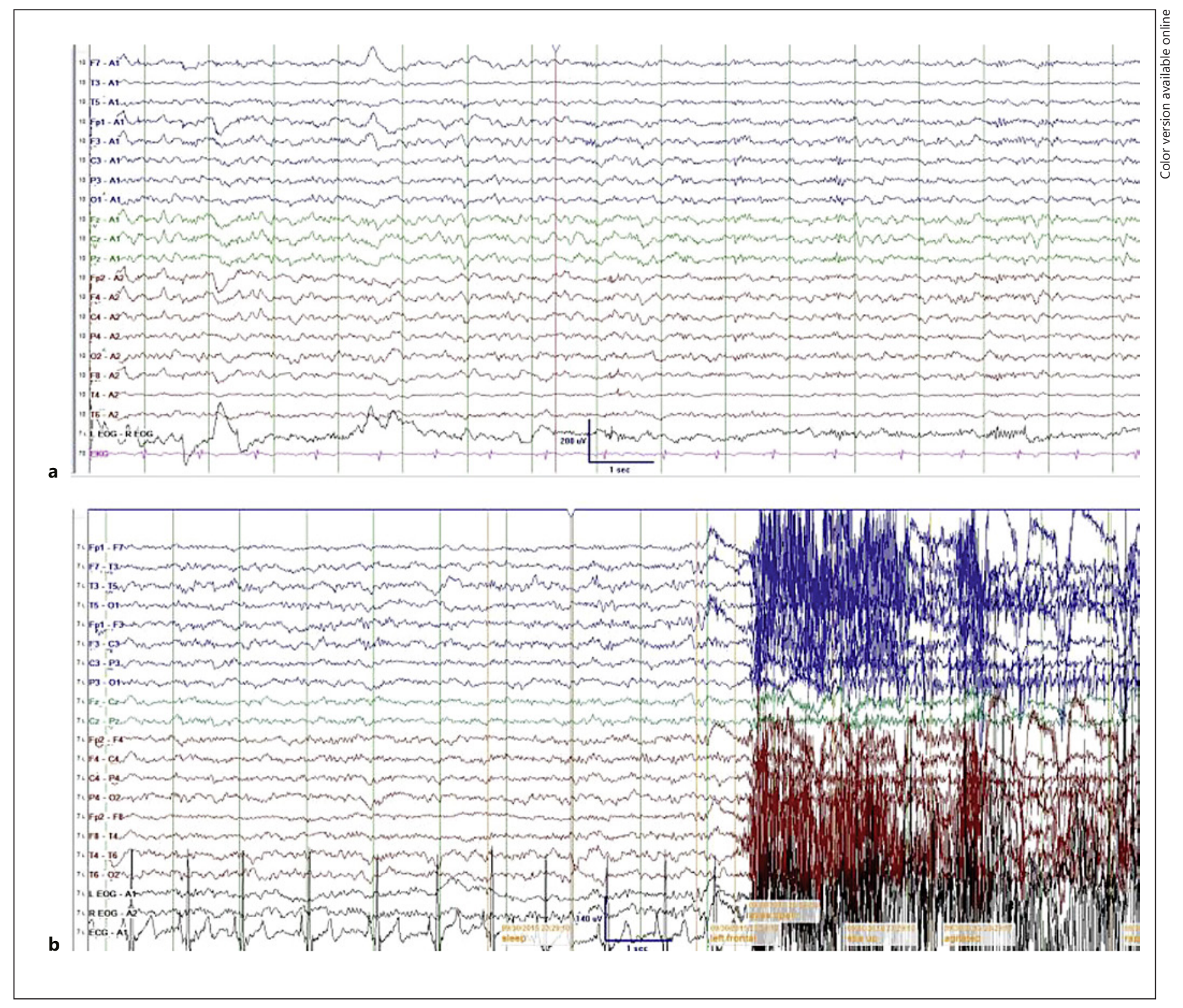

Fig. 1. Both interictal and ictal scalp EEG failed to definitively localize the EZ. Interictal EEG demonstrated bifrontal, left greater than right sharps (a), and ictal EEG (b) demonstrated sometimes left, but predominantly bifrontal sharps followed by artifact.

\section{Postoperative Course}

The patient was discharged home on postoperative day 2 at her neurologic baseline, and she remained seizure free throughout a prolonged AED wean for 8 months postoperatively. After experiencing breakthrough seizures on 1 AED, her seizures are currently intermittent 29 months postoperatively on 2 AED. Repeat noninvasive imaging again failed to localize an EZ; however, given the patients partial response with ablation, repeat robotic-assisted S-EEG was performed and confirmed a right anterior cingulate gyrus EZ. Surgical resection of the ablation zone with wider margins is being considered.
Fig. 2. Imaging workup failed to localize the EZ. An exhaustive imaging workup in this patient was notable for a normal MRI (a) and PET (not shown), with fRMI demonstrating left greater than right activation of receptive and expressive language areas (not shown). MEG demonstrated a first cluster of spikes (90\%) originating from the anterior aspect of the right superior frontal gyrus, but a second group of spikes (10\%) originating from the left frontal operculum and the adjacent superior left insula (b).

(For figure see next page.)
Upadhyayula/Rennert/Hoshide/Sattar/ Gonda 


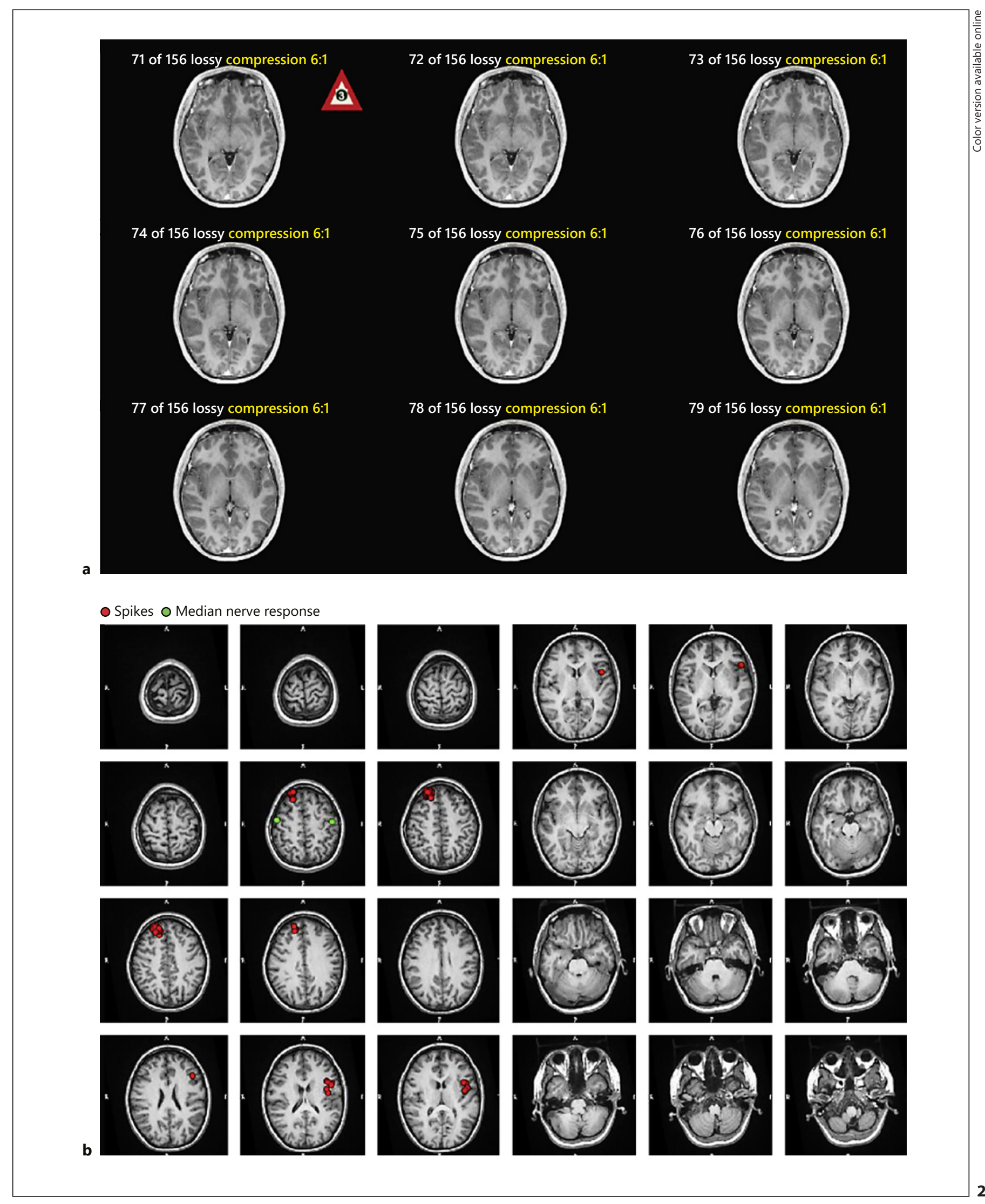

S-EEG and Laser Ablation for Cingulate Gyrus Epilepsy

Stereotact Funct Neurosurg 2019;97:10-17 DOI: $10.1159 / 000496155$ 
Fig. 3. Robotic-assisted S-EEG electrode placement. AP (a) and lateral (b) plain films obtained after placement of 13 S-EEG electrodes, 7 on the left and 6 on the right. Head CT obtained at this time without signs of acute intracranial pathology (images not shown due to electrode-related artifact).
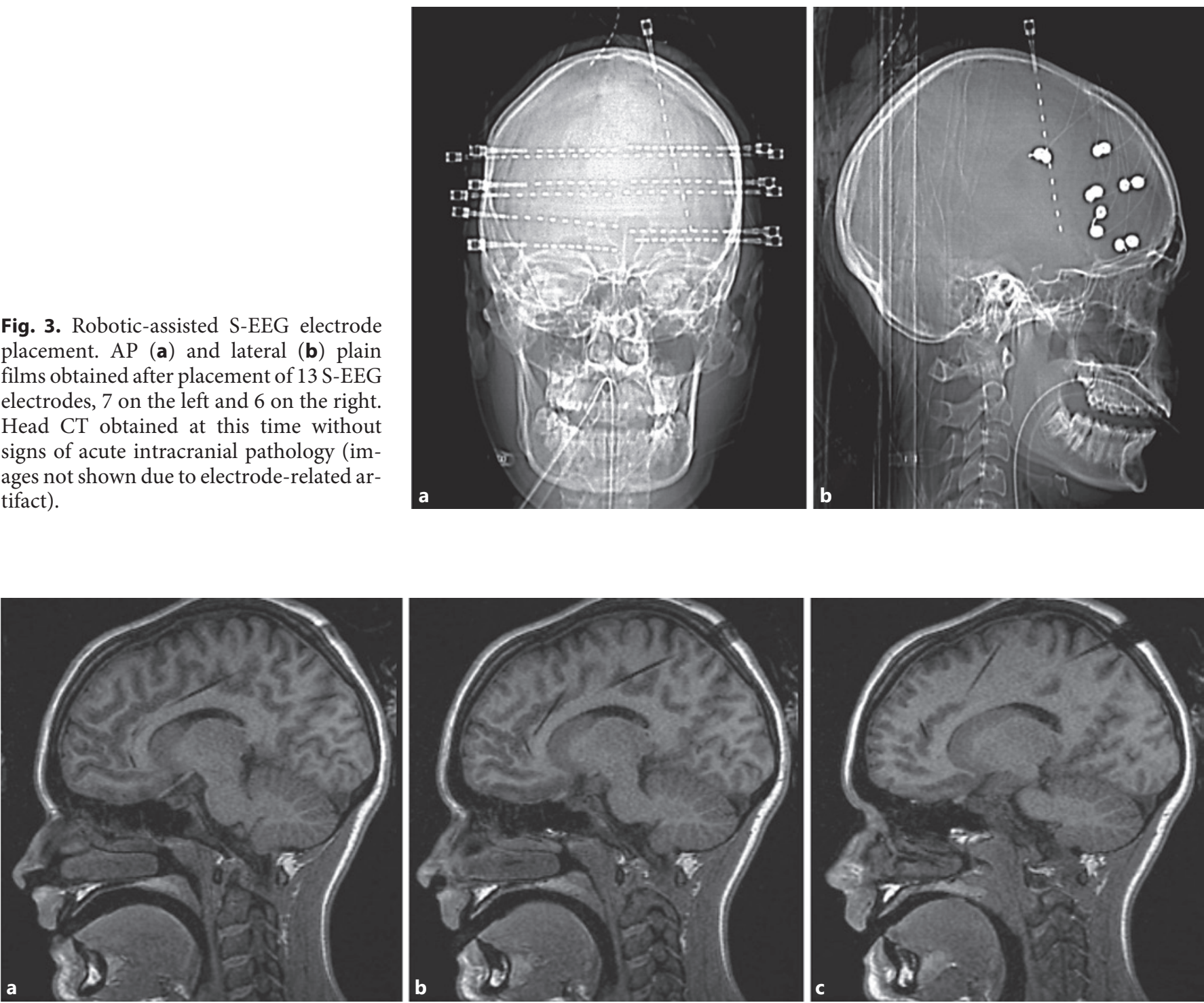

Fig. 4. Robotic-assisted placement of laser ablation leads. Serial views (a-c) of a sagittal T1-weighted MRI confirming placement of ablation leads within the anterior portion of the right cingulate gyrus.

\section{Discussion}

As highlighted by this case report, imaging negative cingulate gyrus epilepsy is a rare pathology that is difficult to diagnose and treat using traditional approaches. Although the patient presented with classic signs of a cingulate gyrus epileptic focus (i.e., fear, hyperkinetic movement), conventional localization studies (fMRI, MRI, PET, and MEG) were suggestive of a frontal lobe EZ but were overall nonspecific. While this was consistent with the patient's mutational status, as familial DEPDC5 mu- tations are often imaging negative, it further complicated her care as surgical resection is typically only recommended in patients with familial DEPDC5 mutations when focal cortical dysplasia is present on imaging [13].

Safety was thus paramount in devising secondary localization and curative strategies in this patient, with a focus on minimally invasive techniques that were both suited to her likely deep EZ and novel in their application within the neurosurgical literature. S-EEG was chosen for EZ localization, as the morbidity rate associated with SEEG is approximately $0.18 \%$ per electrode [14]. While the
Upadhyayula/Rennert/Hoshide/Sattar/ Gonda 

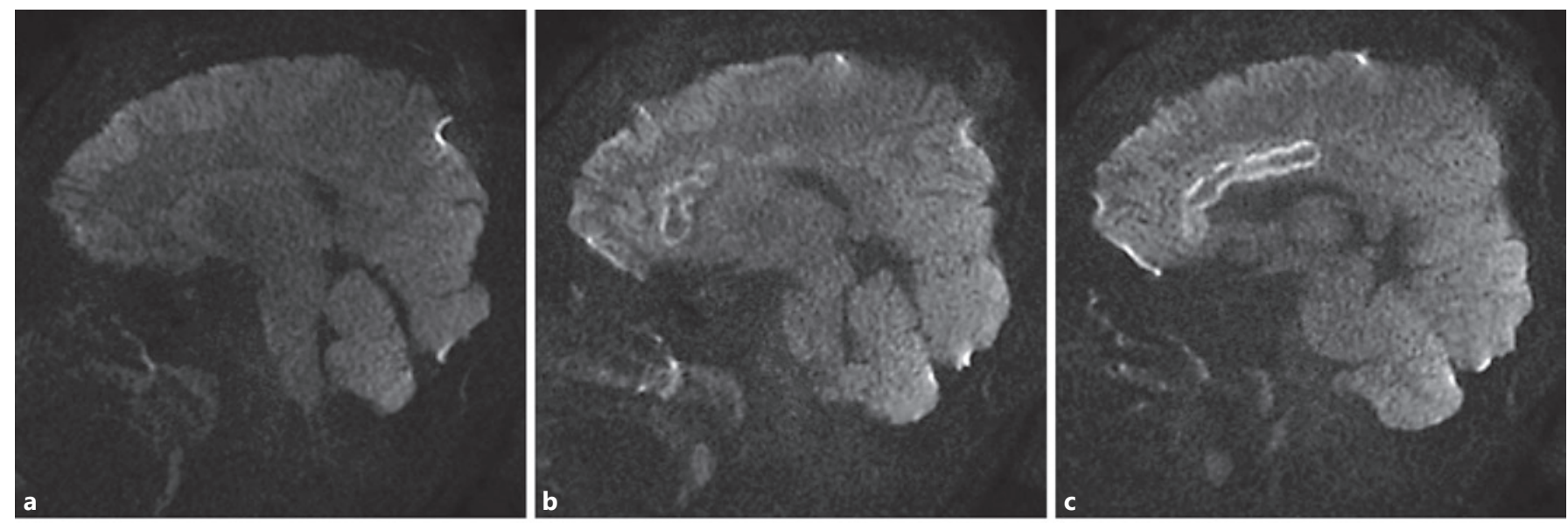

Fig. 5. Successful focal laser ablation of cingulate gyrus epileptogenic zone. Serial diffusion weighted sagittal MRI images (a-e) demonstrating focal ablation of the anterior portion of the right cingulate gyrus, without damage to surrounding structures.
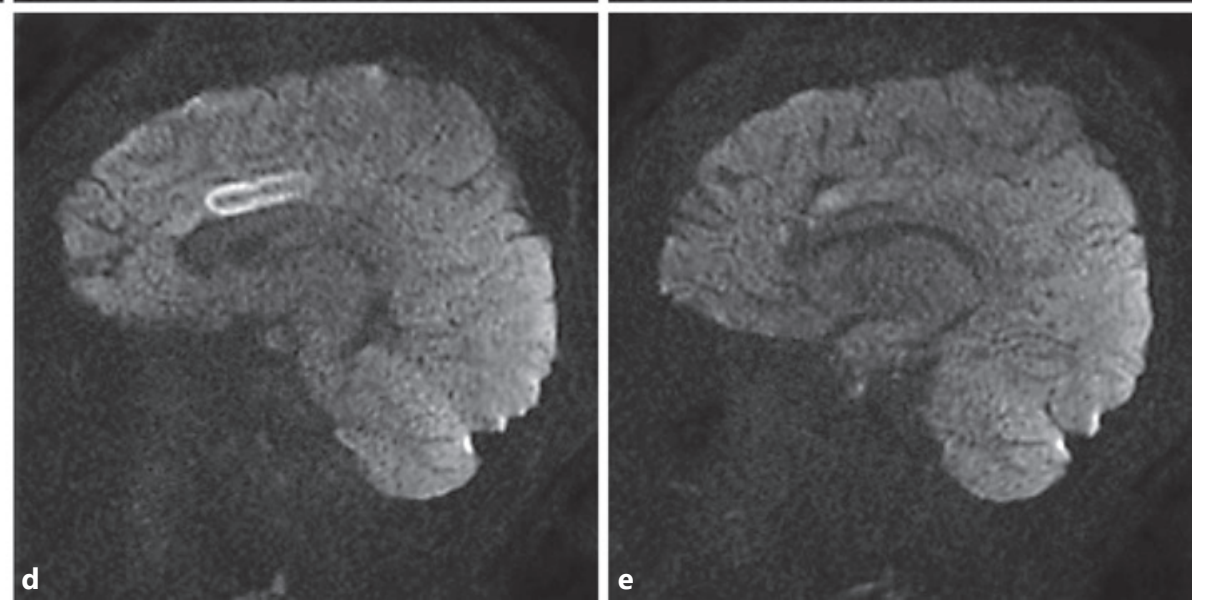

use of S-EEG in pediatric patients is well documented, reports of accurate localization of nonlesional cingulate gyrus EZ using S-EEG are less common [8, 10, 15].

Minimally invasive laser ablation was then chosen for EZ destruction, as its safe use has been described for a multitude of neurosurgical applications, including deep primary/metastatic brain tumors, hypothalamic and intraventricular lesions, and epileptic foci in adult and pediatric patients [16-22]. Data relating to the ablation of eloquent EZ in pediatric patients are nonetheless currently limited to small series [15, 23]. There are only 2 previous reports of laser ablation of cingulate gyrus EZ: one case that was nonlesional (but did not report mutational status or use robotic assistance) [15] and the other resulting from a tuberous sclerosis lesion [23].

Use of the surgical robot for both S-EEG and laser ablation lead placement is an additional unique application of technology within this case. While a relatively new field, robotic-assisted surgeries have been reported across the neurosurgical spectrum, including minimally invasive spine surgery [24], deep brain stimulation [25]), and laser tumor ablation [26]. In the field of epilepsy, however, there is only one report of a robotic-assisted laser ablation of a periventricular EZ in an adult patient [27] and a small series describing use of a robotic system for S-EEG placement in pediatric patients [12]. This latter report also describes a robotic-assisted laser ablation of the posterior corpus callosum for epilepsy in one patient. Use of a robotic system for both placement of S-EEG and ablation leads in the same patient has nonetheless not been described, nor has the application of this technology to cingulate gyrus epilepsy.

The advantages of robotic-assisted S-EEG placement and ablation in this setting are perhaps best appreciated when compared to more traditional, open procedures. For nonlesional epilepsy, congruence across multiple modalities, including scalp EEG, PET, and fMRI, is typically needed before standard invasive neuromonitoring is considered due to the surgical risk of the procedure [6]. 
Then, if the EZ is localized to the cingulate gyrus, the morbidity related to open transcortical or interhemispheric approaches to this area is reported to be as high as $41 \%$ in epilepsy surgery [6] and $89 \%$ in tumor surgery [28], with a nearly $10 \%$ rate of permanent neurologic injury. The improved risk profile of the minimally invasive techniques used in this case thus enabled the safe surgical localization and ablation of at least a portion of the EZ in this patient, who without this technology would likely not have been considered a surgical candidate. Even though the patient experienced a delayed seizure recurrence postoperatively, the partial response with ablation confirmed the relative location of the EZ for a potential future resection of the ablated area with wider margins (with the recurrent seizures shown to be originating from the residual cortex surrounding the ablation on repeat S-EEG despite postoperative noninvasive testing again failing to localize an epileptogenic source).

An additional advantage of robotic assistance in this case is the surgical precision and operative efficiency afforded by robotic assistance. S-EEG and laser ablation often require placement of multiple leads, and when using traditional stereotaxy demand meticulous attention to details throughout a multistep and time-consuming process. The robot allows the user to preplan and preprogram multiple trajectories, and after interface setup allows for a seamless and fast transition between trajecto- ries. In this case, 13 S-EEG electrodes were placed in 165 $\mathrm{min}$ in the first procedure, and 3 Visualase laser ablation catheters were placed in $188 \mathrm{~min}$ in the second procedure. Subsequent cases by the senior author for the expanding indications for this approach have led to progressively shorter operative times for each technique.

\section{Conclusion}

Robotic assistance can facilitate S-EEG and laser ablation of eloquent EZ in pediatric patients with difficult to localize refractory epilepsy.

\section{Statement of Ethics}

The authors have no ethical conflicts to disclose.

\section{Disclosure Statement}

Dr. David D. Gonda has a consulting relationship with Zimmer Biomet. There were no gains, financial or otherwise, related to the production of this paper, which was produced independently of Zimmer Biomet. All other authors certify that they have no disclosures or conflicts of interest to report.

\section{References}

1 Kessler RC, Lane MC, Shahly V, Stang PE. Accounting for comorbidity in assessing the burden of epilepsy among US adults: results from the National Comorbidity Survey Replication (NCS-R). Mol Psychiatry. 2012 Jul; 17(7):748-58.

2 Ficker DM, So EL, Shen WK, Annegers JF, O’Brien PC, Cascino GD, et al. Populationbased study of the incidence of sudden unexplained death in epilepsy. Neurology. 1998 Nov;51(5):1270-4.

3 Iwasaki M, Jin K, Nakasato N, Tominaga T. Non-invasive Evaluation for Epilepsy Surgery. Neurol Med Chir (Tokyo). 2016 Oct; 56(10):632-40.

4 Alkawadri R, Mickey BE, Madden CJ, Van Ness PC. Cingulate gyrus epilepsy: clinical and behavioral aspects, with surgical outcomes. Arch Neurol. 2011 Mar;68(3):381-5.
5 Javidan M. Electroencephalography in mesial temporal lobe epilepsy: a review. Epilepsy Res Treat. 2012;2012:637430

6 von Lehe M, Wagner J, Wellmer J, Clusmann $\mathrm{H}, \mathrm{Kral} \mathrm{T}$. Epilepsy surgery of the cingulate gyrus and the frontomesial cortex. Neurosurgery. 2012 Apr;70(4):900-10.

7 Mirandola L, Meletti S, Cantalupo G. Longterm surgery outcome for epilepsy and psychogenic nonepileptic seizures in a child with anterior cingulate gyrus dysplasia. Epilepsy Behav Case Rep. 2015 Feb;3:20-2.

8 Alkawadri R, Gonzalez-Martinez J, Gaspard N, Alexopoulos AV. Propagation of seizures in a case of lesional mid-cingulate gyrus epilepsy studied by stereo-EEG. Epileptic Disord. 2016 Dec;18(4):418-25.
9 Steiger HJ, Göppert M, Floeth F, Turowski B, Sabel M. Fronto-mesial WHO grade II and III gliomas: specific aspects of tumours arising from the anterior cingulate gyrus. Acta Neurochir (Wien). 2009 Feb;151(2):137-40.

10 Lacuey N, Davila JC, Zonjy B, Amina S, Couce $\mathrm{M}$, Turnbull J, et al. Lesion-negative anterior cingulate epilepsy. Epileptic Disord. 2015 Jun; 17(2):134-42.

11 Gonzalez-Martinez J, Mullin J, Bulacio J, Gupta A, Enatsu R, Najm I, et al. Stereoelectroencephalography in children and adolescents with difficult-to-localize refractory focal epilepsy. Neurosurgery. 2014 Sep;75(3):258-68.

12 Miller BA, Salehi A, Limbrick DD Jr, Smyth MD. Applications of a robotic stereotactic arm for pediatric epilepsy and neurooncology surgery. J Neurosurg Pediatr. 2017 Oct;20(4): $364-70$.
16

Stereotact Funct Neurosurg 2019;97:10-17 DOI: $10.1159 / 000496155$
Upadhyayula/Rennert/Hoshide/Sattar/ Gonda 
13 Baulac S, Weckhuysen S. DEPDC5-Related Epilepsy. In: Pagon RA, Adam MP, Ardinger $\mathrm{HH}$, Wallace SE, Amemiya A, Bean LJ, et al., editors. GeneReviews $\left({ }^{\circledR}\right)$. Seattle (WA): University of Washington; 2016.

14 Gonzalez-Martinez J, Mullin J, Vadera S, Bulacio J, Hughes G, Jones S, et al. Stereotactic placement of depth electrodes in medically intractable epilepsy. J Neurosurg. 2014 Mar; 120(3):639-44.

15 Marashly A, Loman MM, Lew SM. Stereotactic laser ablation for nonlesional cingulate epilepsy: case report. J Neurosurg Pediatr. 2018 Nov;22(5):481-8.

16 Ali MA, Carroll KT, Rennert RC, Hamelin T, Chang L, Lemkuil BP, et al. Stereotactic laser ablation as treatment for brain metastases that recur after stereotactic radiosurgery: a multiinstitutional experience. Neurosurg Focus. 2016 Oct;41(4):E11.

17 Buckley RT, Wang AC, Miller JW, Novotny EJ, Ojemann JG. Stereotactic laser ablation for hypothalamic and deep intraventricular lesions. Neurosurg Focus. 2016 Oct;41(4):E10.
18 Jermakowicz WJ, Kanner AM, Sur S, Bermudez C, D'Haese PF, Kolcun JP, et al. Laser thermal ablation for mesiotemporal epilepsy: analysis of ablation volumes and trajectories. Epilepsia. 2017 May;58(5):801-10.

19 Rennert RC, Carroll KT, Ali MA, Hamelin T, Chang L, Lemkuil BP, et al. Safety of stereotactic laser ablations performed as treatment for glioblastomas in a conventional magnetic resonance imaging suite. Neurosurg Focus. 2016 Oct;41(4):E7.

20 Rolston JD, Chang EF. Stereotactic Laser Ablation for Hypothalamic Hamartoma. Neurosurg Clin N Am. 2016 Jan;27(1):59-67.

21 Thomas JG, Rao G, Kew Y, Prabhu SS. Laser interstitial thermal therapy for newly diagnosed and recurrent glioblastoma. Neurosurg Focus. 2016 Oct; 41(4):E12.

22 Rennert RC, Khan U, Tatter SB, Field M, Toyota B, Fecci PE, et al. Patterns of Clinical Use of Stereotactic Laser Ablation: Analysis of a Multicenter Prospective Registry. World Neurosurg. 2018 Aug;116:e566-70.

23 Curry DJ, Gowda A, McNichols RJ, Wilfong AA. MR-guided stereotactic laser ablation of epileptogenic foci in children. Epilepsy Behav. 2012 Aug;24(4):408-14.
24 Lefranc M, Peltier J. Evaluation of the ROSA $^{\mathrm{TM}}$ Spine robot for minimally invasive surgical procedures. Expert Rev Med Devices. 2016 Oct;13(10):899-906.

25 Vadera S, Chan A, Lo T, Gill A, Morenkova A, Phielipp NM, et al. Frameless Stereotactic Robot-Assisted Subthalamic Nucleus Deep Brain Stimulation: Case Report. World Neurosurg. 2017 Jan;97:762.e11-4.

26 Brandmeir N, Acharya V, Sather M. Robot Assisted Stereotactic Laser Ablation for a Radiosurgery Resistant Hypothalamic Hamartoma. Cureus. 2016 Apr;8(4):e581.

27 Gonzalez-Martinez J, Vadera S, Mullin J, Enatsu R, Alexopoulos AV, Patwardhan R, et al. Robot-assisted stereotactic laser ablation in medically intractable epilepsy: operative technique. Neurosurgery. 2014 Jun;10 Suppl 2: $167-72$.

28 von Lehe M, Schramm J. Gliomas of the cingulate gyrus: surgical management and functional outcome. Neurosurg Focus. 2009 Aug; 27(2):E9. 